the system of another - superficially very different - one. Most TAU's are named by common adages, such as "a stitch in time saves nine", "too many cooks spoil the broth", and "many hands make light work". These are defined as patterns of planning and plan-adjustment which can be instantiated by many examples; associations within the program's semantic memory allow one example of "saving nine" to recall another.

The author's intent is to provide a psychological simulation of parsing and memory organization, and some experiments are cited accordingly. However, the psychological similarities may not go very deep. That stories are classified as "similar" with respect to their thematic nature (not their specific content) is a result which suggests that something broadly like TAU's are functioning within human

\section{Point to point}

\section{C.J.S. Clarke}

The Genesis and Evolution of Time: $A$

Critique of Interpretation in Physics.

By J.T. Fraser.

Harvester Press/University of

Massachusetts: 1983. Pp.193. £19.50,

$\$ 20$.

The Enigma of Time.

By P.T. Landsberg.

Adam Hilger, Bristol: 1982. Pp.248.

Hbk £13.95, \$28; pbk £7,95.

Is THERE such a thing as 'the riddle of time', whose solution would explain 'life, the universe and everything'? Or does there in fact exist a single phenomenon going under the name of time? It is the contention of J.T. Fraser that Time does constitute a unified subject of paramount importance, but that time is multiple and not simple.

His thesis is that the world of which we have knowledge can be thought of as organized into a hierarchy of levels, to each of which different laws of nature are appropriate, and in each of which time has a different character. Only at the human level does time have all the properties of a continuous past/present/future succession. The properties become progressively more rarified as one progresses to levels remote from our own, until at the lowest, most microscopic level, claims Fraser, there remains no time at all.

He realizes, of course, the need to show that this hypothesis can be made sufficiently precise for it to be tested against the actuality of nature. But unfortunately it is often hard to tell whether this approach really scores over more conventional ones: partly because he never enters into a detailed point-by-point critique of the alternative views; partly because the level of presentation is always very general, eschewing detailed mathematical analysis at every stage; and partly I suspect, because memory. But how broad is "broad"?

Only Boris, of course, could really understand one's diary: BORIS has no capacity for empathy or fellow-feeling. The program's "understanding" consists merely in manipulating symbols including English words - so as to draw what human beings can interpret as sensible inferences about the interpersonal relations mentioned in the narrative. And only Boris could be bribed, with a suitable tincture, to use his discretion in passing on personally sensitive information. The teetotal BORIS's uncensored output might prove highly embarrassing if relayed to the Moscow end of a telephone wire. Perhaps Denis Thatcher should be thankful for small mercies.

Margaret Boden is Professor of Philosophy and Psychology at the University of Sussex.

he has chosen to ignore some aspects of the conventional physical view.

Consider, for example, the lowest, atemporal level (one of his most interesting ideas). As his starting point he takes the 'core' aspect of time to be metrical time, in a generalized sense. Time is that which is measured by a process of time-keeping, and time-keeping involves correlating events with some standard series of events chosen so as to yield a simple account of the phenomena. This is the tradition of timeexplication started by Aristotle, beginning with the "numbering of movement" and then looking for a standardization of the numbering. It is sharply at variance with the approach of Newton-Smith, for example, who distinguishes topological time, a linear continuum without metrical properties, as the most basic level of structure for time. On this latter view one should separate the topology of time (involving questions of betweenness and of global topology) from the question of what metric one should place on a time with a given topology.

Fraser derives his lowest level from the consideration of null goedesics in special relativity. Such a geodesic has no intrinsic metric, but it has a perfectly good topology which coincides with the topological time of any inertial observer. And so it should make sense to see a photon as possessing its private topological time, though devoid of metrical time. For Fraser, however, time is metrical in essence and so the photon is timeless and inhabits, with any other massless particles, the atemporal level at the bottom of the hierarchy. The argument thus hinges on a philosophical issue as to the nature of time, where the alternative view (topological time) is not analysed at all.

The atemporal level is supposed to be the level in which the universe originated. From the point of view of cosmology this level lies at the temporal beginning of the universe, although strictly speaking it is without time and so cannot actually partake in a temporal relation. In placing the atemporal level at the big bang, Fraser seems to take it for granted that in its earliest stages the universe was inhabited purely by photons, apparently ignoring the theories, now predominant, that place hadron physics (or quarks etc.) at the start.

On the central queston of the directionality of time, the conventional dichotomy between laws (time-symmetric) and contingent conditions (imparting directionality) is rejected. Each level of nature has to be treated in its own right, and time is to be regarded as directional at that level if it is the case that the occurrence of a system obtained by reversing the time-sense of a normal system is not only unobserved, but is basically impossible. This, he claims, happens only at the biological level, where genetic programming and growth involve an unshakable directionality in time.

But what then is the relation, if any, between the basic time-asymmetry of the biological world and the contingent asymmetries of the physical world? It is acknowledged that the biological asymmetry has its ultimate root in the expansion of the universe, but the account of the link between the two is limited. For instance, although the paper of Penrose, arguing that the asymmetry is reflected in a progressive increase in the Weyl tensor which makes the Big Bang essentially different from the final Big Crunch, is cited, its conclusion is baldly denied by Fraser, who treats Bang and Crunch as equivalent.

Despite these drawbacks, the book has many attractive features. The metaphysics of his levels (his "umwelt principle") is a promising philosophical idea; the picture of an underlying atemporal world, notwithstanding its doubtful derivation, fits well with some speculative attempts to derive space-time from something more primitive in an attempt to avoid the nonrenormalizability of quantum gravity; and the account of the origin of life, of primitive organisms as "clock shops" is intriguing. Fraser may well turn out to be right.

For an introduction to the conventional wisdom, particularly on the physical side, one could hardly better Landsberg's admirable anthology - The Enigma of Time - a selection of reprinted papers on irreversibility, cosmology, quantum theory and black holes with a concluding section on time in art and literature. Landsberg provides a lucid introduction, glossary and indices so as to make the book as accessible as possible to a wide readership. Inevitably, space prevents the detailed development of the themes; one's appetite is whetted for many more papers on the quantum measurement problem and its relation to irreversibility.

The anthology raises many questions, and Fraser's book attempts an answer. It is to be hoped that subsequent works will supply the critical comparisons that are needed to carry the debate further.

Dr C.J.S. Clarke is a Lecturer in Mathematics in the University of York. 